\title{
Biomechanics
}

\section{Dynamics of human movement}

\author{
Bart (H.F.J.M.) Koopman* \\ Department of Biomechanical Engineering, University of Twente, P.O. Box 217, 7500 AE Enschede, \\ The Netherlands \\ E-mail: h.f.j.m.koopman@utwente.nl
}

\begin{abstract}
The part of (bio)mechanics that studies the interaction of forces on the human skeletal system and its effect on the resulting movement is called rigid body dynamics. Some basic concepts are presented: A mathematical formulation to describe human movement and how this relates on the mechanical loads acting on the skeletal system. These equations of motion depend on the mechanical properties of the skeletal system, such as dimensions and mass distribution. It is applied to describe and analyze human gait.
\end{abstract}

Keywords: Human movement, rigid body dynamics, gait analysis

\section{Introduction}

Human movement dynamics relates the applied forces or moments of force (i.e. the kinetics) to the movement (i.e. kinematics) of the skeletal system. In the control loop to displace body parts (Fig. 1), the Central Nervous System (CNS) is the controller that activates the motors (muscles). The movement dynamics are represented by the skeletal system, and the resulting movement is measured and fed back into the CNS.

In this chapter we focus on the skeletal system only. The part of (bio)mechanics that studies this is called rigid body dynamics. Some basic concepts are presented: A mathematical description of human movement; how this depends on the mechanical loads acting on the skeletal system and how this depends on the mechanical properties of the skeletal system, such as dimensions and mass distribution. It will be applied to describe human walking.

\section{Rigid body dynamics}

The human body consists of a more or less rigid structure (the bones of the skeleton) to which soft tissues (muscles, fat, organs etc.) attach. A structure is considered rigid when under practical loading situations, the structure does not noticeably deform, i.e. the mechanical stiffness seems to be infinite. Since the soft tissues are not rigid and do deform in practical loading situations, an accurate mechanical description of human movement involves the description of both soft tissues and skeleton. The description of the dynamics of the human body would involve very complex calculations, since not only movement, but also deformations have to be considered. This approach is only feasible in some very specific applications, for example to study the effect of car crash impacts on brain damage. As it 


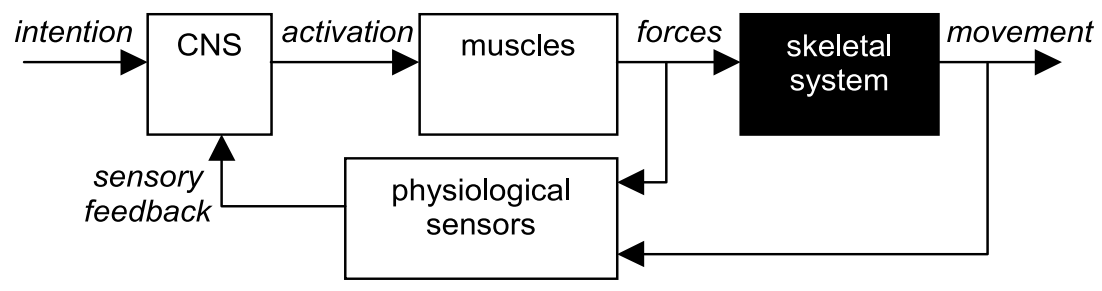

Fig. 1. Schematic block diagram of the human motor control system.

is not possible to measure all soft tissue deformations, some assumptions have to be made to be able to study human movement.

The most important assumption in rigid body dynamics is that movement only occurs in the joints. As a consequence of this assumption, the structures in-between the joints, the so-called segments, are assumed to be rigid, so they have an infinite large stiffness. All passive soft tissue structures are considered to be rigid as well. The muscles are replaced by the forces they produce. So it is assumed that the human body behaves like a segments model or a linked system of rigid bodies. Apart from the enormous reduction of complexity, this viewpoint has other advantages as well: The rigid body equations of motion, when applied to each segment, are sufficient to describe the dynamics of the entire system.

Rigid body dynamics describes the kinematics (movement) and kinetics (forces) of a given segments model. The equations of motion are the link between these two, which is a set of two first-order nonlinear differential equations for each movement component (or, alternatively, a single second order equation that includes a dependency on the second time derivative, the acceleration). For a given segments model the forces and movements provide interchangeable information: The forces can be calculated when the movements are known (i.e. inverse dynamics approach) and the movements can be calculated when the forces are known (i.e. direct or forward dynamics approach).

\section{Kinematics}

\subsection{Medical motion description}

Two bones can move with respect to each other by virtue of the joint in between. In the human body, only the synovial joints allow for large movements. In principle, a bone has six Degrees-of-Freedom (DOF) of motion with respect to the other bone: three rotations and three translations. The motions of the joint are limited by passive structures like the articular surfaces and the ligaments. These passive structures pose restraints to the joint motions: Though motions in the direction of the restraint are still possible, these motions will be very small.

For example, cartilage can be compressed a few millimeters. Most of the time these small motions are neglected, the motion is said to be constrained. For each constraint the number of DOF diminishes by one. As soon as constraints come into the picture, one has already started modeling the joint. In Fig. 2 all combinations of (constrained) rotations and translations are shown. Many of them are merely hypothetical, and will not be found in the human body.

Traditionally, joints have been studied (e.g. by Fick [4]) by comparing them with standard revolute joints: Hinges, spherical joints, ellipsoidal or condyloidal joints, saddle joints (see Fig. 3). The shoulder joint and the hip joint behave approximately as spherical joints only permitting three rotational DOF. The elbow joint and finger joints can be regarded as hinge joints with one rotational DOF. The 


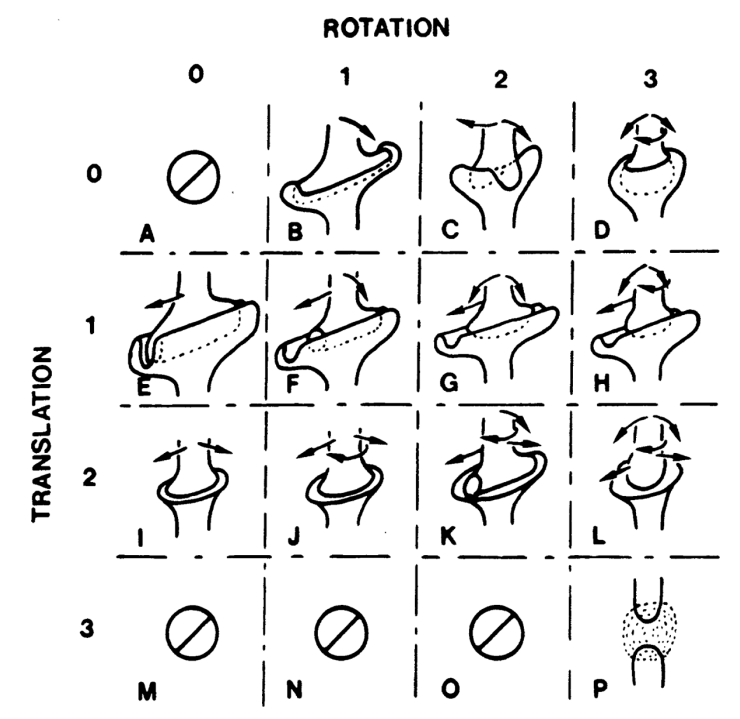

Fig. 2. Combinations of rotations and translations in joints. Many joints shown are hypothetical and are not found in the human body.

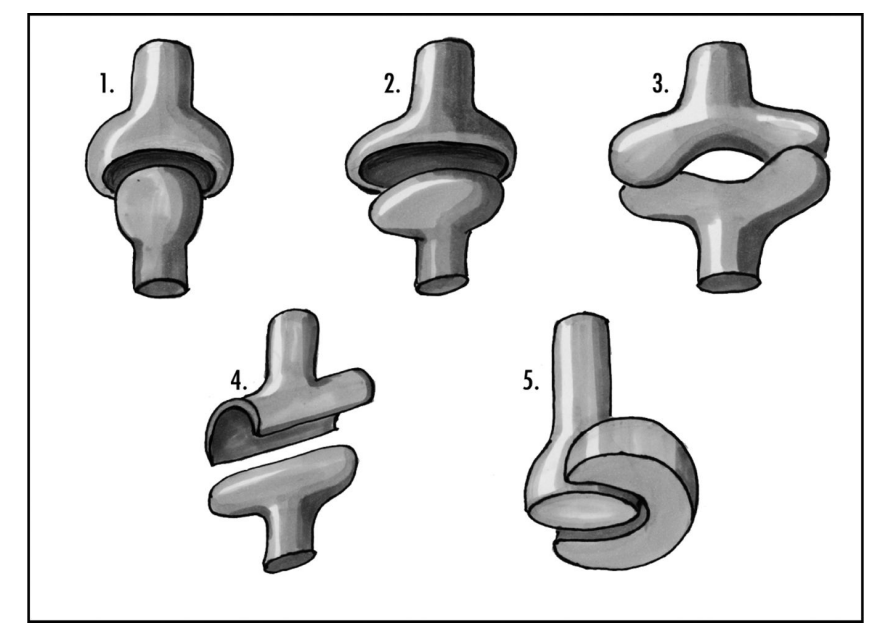

Fig. 3. Schematic drawing of 5 joint types, ball and socket (1), condyloid (2), saddle (3) hinge (4) and pivot joints (5).

first metacarpo-phalangeal (thumb) joint and the ankle joint resemble a saddle joint, having two nonintersecting rotational axes. But often one cannot derive the potential motions from the shape of the articular surfaces.

Since anatomists were the first to study joint motions, medical definitions still dominate the way joint motions are described. The goal of such medical definitions is to distinguish between pathological and normal motion, and to evaluate the outcome of treatment: Is there improvement in the range of motion or not.

As a starting position the anatomical position (Fig. 4) is used. From this position the motion is defined for each single rotation. For spherical joints, the rotation axes are defined along the axis of the global coordinate system: A vertical axis and two horizontal axes, pointing backward-forward 


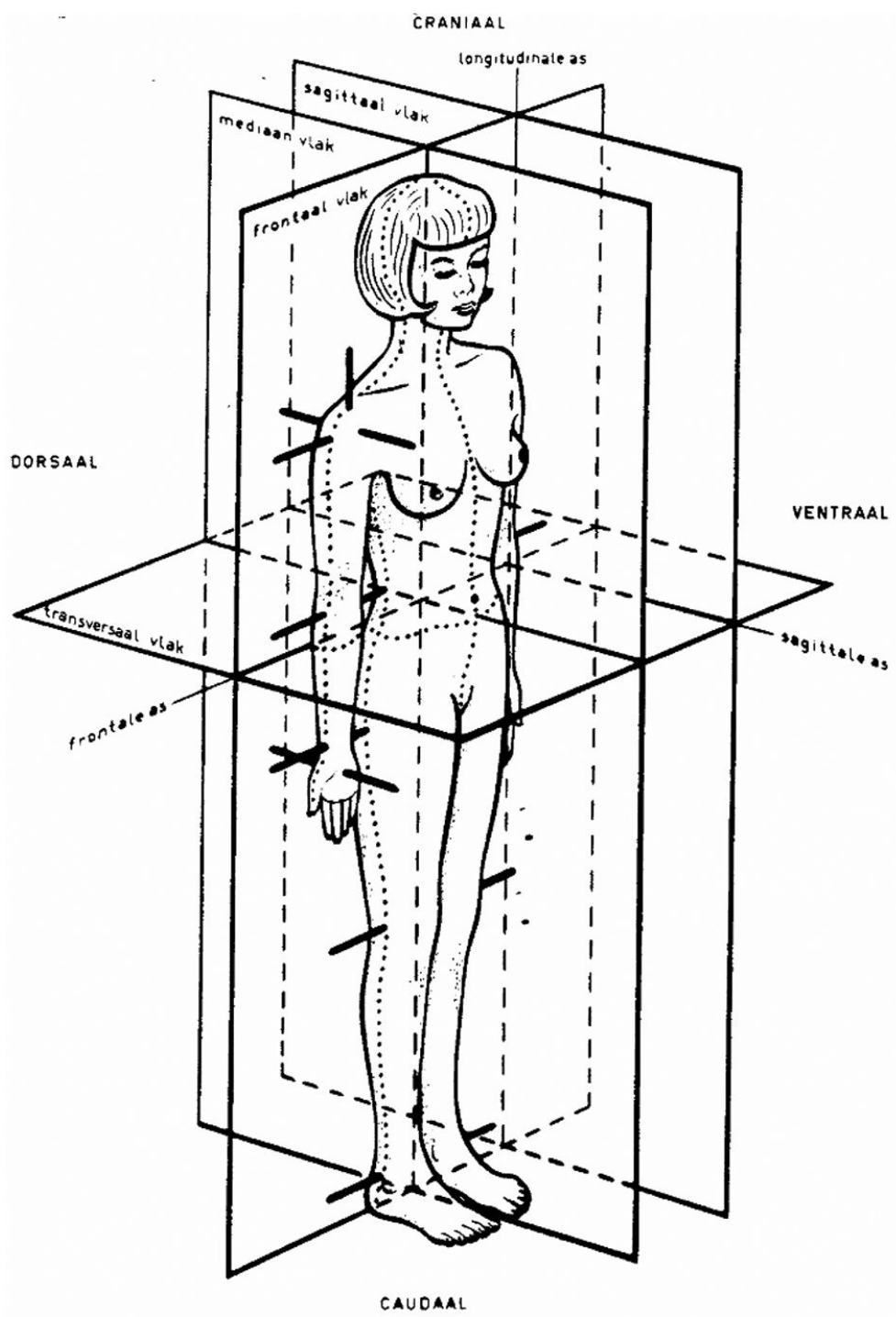

Fig. 4. The anatomical positions.

(antero-posteriorly) and side-side (medio-laterally, from the centre of the body to the left or right side). Commonly used terms are flexion-extension, abduction-adduction, and medial and lateral rotation. A problem occurs if the motion is not about just one of the standardized axes, but is a combination of rotations. The order of rotations is not defined, and it is not clear whether the rotation axes move with the bone or not. This causes much confusion about rotation angles, and makes comparison between studies often impossible.

\subsection{The segments model}

All models to analyze or simulate human movement are based on segments. These are coupled systems of rigid bodies, with dimensions and inertial properties that are deduced from the dimensions 

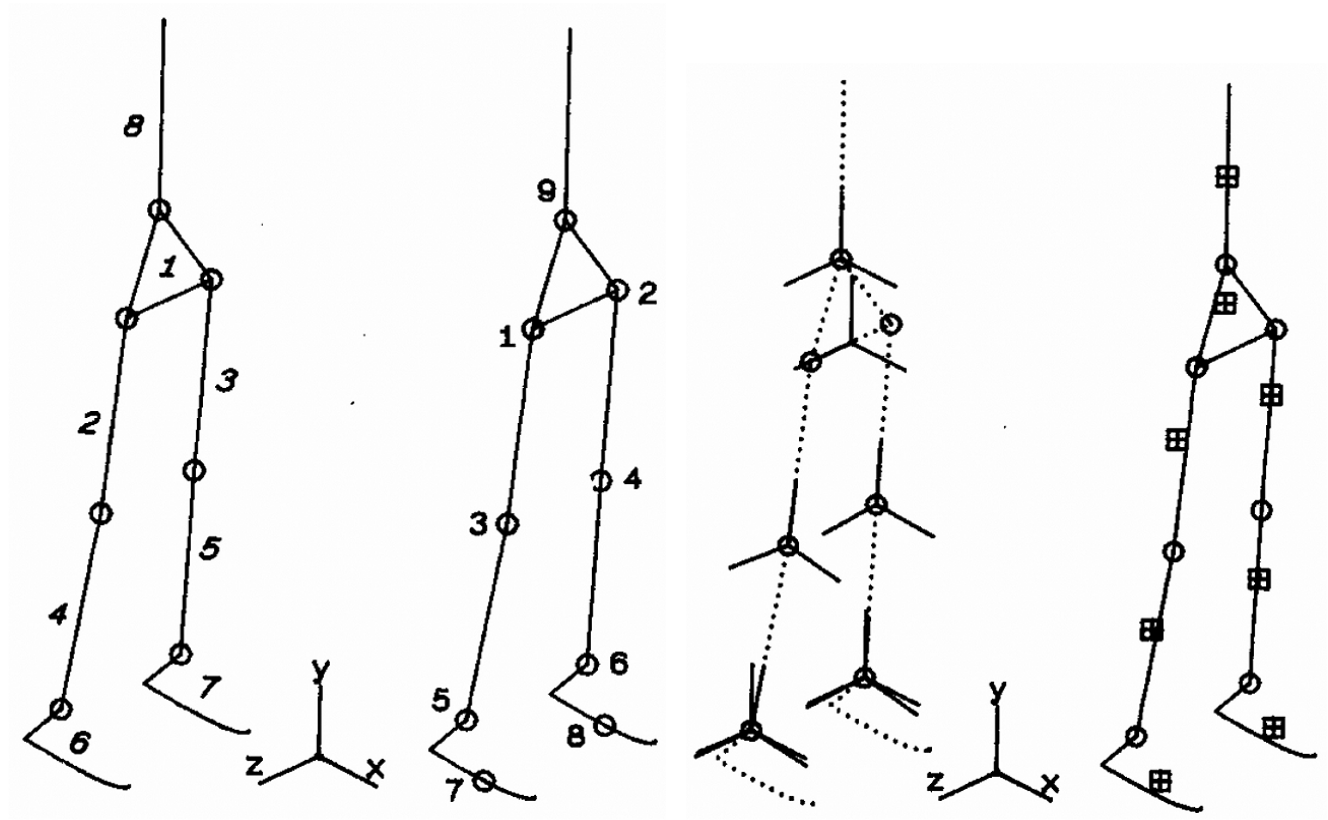

Fig. 5. The segments model for the bady at rest. From left to right: Numbering of segments and joints, definition of the reference frame, definition of local frames and positions of the centers of mass.

and inertial properties of the human body. Initially, these segments models were used to dimension the dummies used in car crash experiments [3] and were therefore based on averaged data of human bodies. With the introduction of the segments models to computer simulations, a wider variety became possible. The segments models became scalable, with inertial properties usually depending on local segment dimensions and total body weight. In this way, the segments model could be matched to fit each individual [2].

The choice of the number of segments should be large enough to simulate the movement adequately. Too large a number of segments, however, would lead to unnecessary complexity and larger computational efforts. To simulate walking, segments models varying from 3 segments [10] up to 17 segments [6] have been proposed. To further reduce complexity, symmetry between right and left leg is often assumed [1] and the movement is often restricted to the sagittal plane only.

In a model for normal walking, there are segments for the thighs, shanks and feet. The head, arms and trunk (HAT) and the pelvis are modeled as two separate segments. The segments are connected to each other at the joints (Fig. 5). Although it is possible to have more than two joints in a segment (e.g. the pelvis), each joint is connecting just two segments. To define the position of the segments in space, an absolute or reference frame is attached to the floor, with the $\mathrm{x}$-axis pointing in the walking direction, the $y$-axis pointing upward and the z-axis perpendicular to the xy-plane in the lateral direction. Figure 5 shows an 8-segmental model with numbering of the segments, the joints and definition of the reference frame. It should be noted that the shape of the segments is of no importance as long as the positions of the joints and the mass properties are well defined. In each segment, a local frame is defined, standardized with the help of some bony landmarks on the segment.

The segments model must include the following mechanical properties, which are dependent on individual characteristics: The dimensions and positions of the segments in space, the positions of the joints, and the mass properties of the segments (mass, position of the center of mass and moment of 


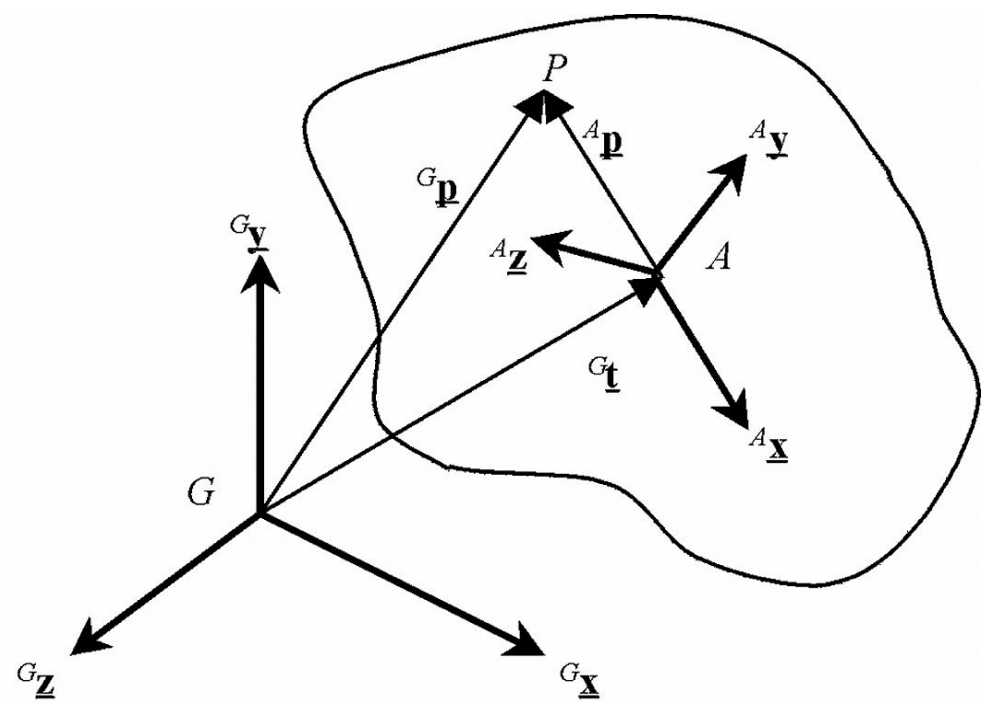

Fig. 6. Transformation of position vector ${ }^{A} \mathbf{p}$ from local coordinate system A to position vector ${ }^{G} \mathbf{p}$ in the global coordinate system $\mathrm{G}$.

inertia tensor). Other properties, such as the ranges of motion of the joints, the maximal moments of force that can be exerted on the joints, and muscle models, may also be included [8].

\subsection{Mathematical motion description}

The segments model is the basis for a sound technical description of human movement. In general, a movement of a rigid body is defined as the displacement and orientation of the local frame relative to the reference frame or another local frame. Therefore, any description of a (body or joint) rotation implicitly assumes that some kind of segments model is defined, otherwise the rotation has no unique meaning. The anatomical position is usually taken as the offset position for the segments model where the relative rotations are defined as zero.

Mathematically, the motion of a body with respect to another body is described unambiguously by a [3x3] rotation matrix $\mathbf{R}$ and a translation vector $\mathbf{t}$ [5], see Fig. 6 . This results in a linear equation for an arbitrary position vector $\mathbf{p}$. The rotation matrix can be viewed as some multi-dimensional function that, when it operates on the vector $\underline{\mathbf{p}}$, it changes the orientation of $\underline{\mathbf{p}}$. Note that $\underline{\mathbf{p}}$ has 3 components, and each component may change with respect to each of the 3 dimensions, so the rotation matrix represents 9 (or [3x3]) dependencies.

$$
\begin{aligned}
& \left({ }^{G} \mathbf{p}-{ }^{G} \underline{\mathbf{t}}\right)={ }^{G A} \mathbf{R} \cdot\left({ }^{A} \underline{\mathbf{p}}-{ }^{A} \underline{\mathbf{t}}\right) \\
& { }^{G} \underline{\mathbf{p}}={ }^{G} \underline{\mathbf{t}}+{ }^{A} \mathbf{R} \cdot{ }^{A} \underline{\mathbf{p}}
\end{aligned}
$$

Bold capitals are used to denote matrices; underlined lowercase characters are used to denote vectors. ${ }^{G} \mathbf{p}$ is the position vector of point $P$ in the global coordinate system $G,{ }^{A} \mathbf{p}$ is the position vector of point $P$ in the local coordinate system $A .{ }^{G} \underline{\underline{\mathbf{t}}}$ is the translation vector from the origin of $A$ expressed in coordinate system $G$.() Obviously ${ }^{A} \underline{t}$, the origin of $A$ expressed in coordinates of $A$ equals the zero vector $\underline{\mathbf{0}}{ }^{G A} \mathbf{R}$ is the rotation matrix that describes the rotation from the local coordinate system $A$ to the coordinate system $G$ (the global coordinate system). 
Element-wise, equation (1) looks like

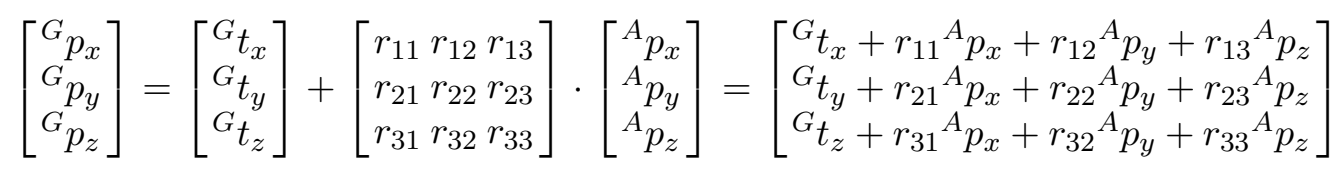

in which the elements of the rotation matrix are cosines of the angles between the axes of the global and local coordinate system (see Fig. 6):

$$
\begin{aligned}
& { }^{G A} \mathbf{R}=\left[\begin{array}{lll}
r_{11} & r_{12} & r_{13} \\
r_{21} & r_{22} & r_{23} \\
r_{31} & r_{32} & r_{33}
\end{array}\right]=\left[\begin{array}{l}
\cos \left(\theta_{11}\right) \cos \left(\theta_{12}\right) \cos \left(\theta_{13}\right) \\
\cos \left(\theta_{21}\right) \cos \left(\theta_{22}\right) \cos \left(\theta_{23}\right) \\
\cos \left(\theta_{31}\right) \cos \left(\theta_{32}\right) \cos \left(\theta_{33}\right)
\end{array}\right] \\
& \theta_{11}=\angle\left({ }^{A} \underline{\mathbf{x}},{ }^{G} \underline{\mathbf{x}}\right) \text {, } \\
& \theta_{21}=\angle\left({ }^{A} \underline{\mathbf{x}},{ }^{G} \underline{\mathbf{y}}\right), \quad \text { etc. }
\end{aligned}
$$

Although the rotation matrix contains 9 elements, there exist dependencies between them because we assume rigidity of the segment it represents. Ultimately, there are only three independent variables present. Together with the three elements of the translation vector, these six variables describe the potential 6 DOF motion of the segment or between two bones. To better visualize motion and comparison between subjects, the rotation matrix is reduced to three successive rotations about well-defined axes, so-called Euler angles. This can be viewed as three hinge joints in succession. Rotation of the first hinge will change the orientation of the second and third, rotation of the second hinge will change the orientation of the third hinge, and rotation about the third hinge will result in the desired orientation of the local coordinate system.

In Biomechanics, usually Cardan angles are used, which are Euler angles with successive rotations about the local $x$-, $y$ - and $z$-axes. Instead of the order $x-y-z$, any other combination of these rotations could have been chosen. Since matrix multiplication is not a commutative operation (a different order of the rotations, e.g. $y-x-z$ instead of $x-y-z$, will give different outcomes), each combination of rotations will result in other values of the angles. Other parameterizations for the orientation may be used as well. For example, a well-known parameterization for rigid body movement is the Finite Helical Axis (FHA) definition, which utilizes helical angles.

\section{Dynamics}

The next step after defining the movement of rigid bodies is to derive the equations of motion that define the relations between the acting forces and torques and the movement. A straightforward way to do this is to apply the Newton-Euler approach. Although alternative formulations exist that have specific applications, such as the Lagrange approach, the Newton-Euler approach is simplest because:

The method is identical for each segment.

It is based on the Free Body Diagram of the segment. With this Free Body Diagram there is a simple recipe to obtain the equations of motion.

The equations of motion for the entire body is the sum of the equations for each segment.

Newton formulated the equations of motion for systems of mass particles. Euler recognized that a rigid body is a special case for such a system: The positions of the particles are constrained with respect to each other. This leads to the notion that the internal forces (the forces acting between the particles) do 


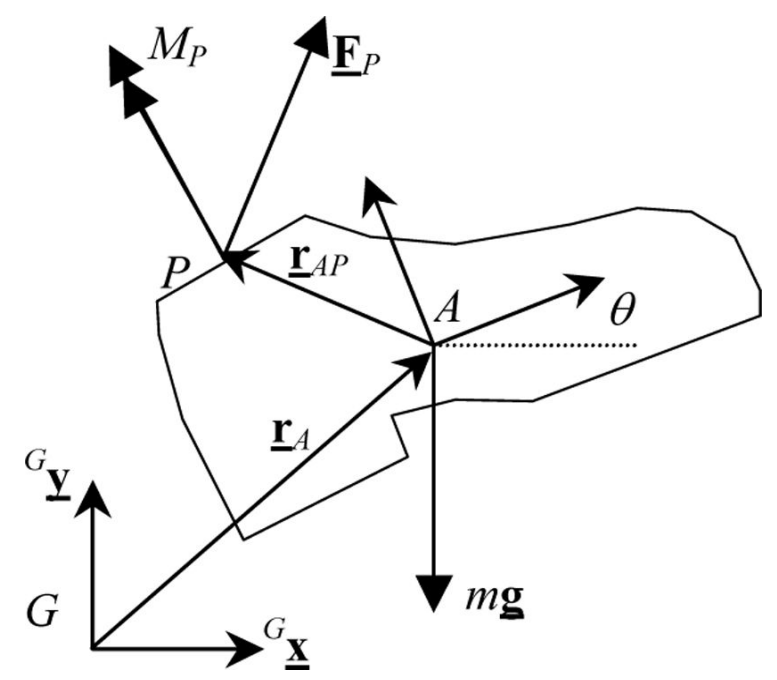

Fig. 7. Free body diagram of a rigid body A with joint $\mathrm{P}$.

not perform work and do not contribute to the equations of motion for the entire system, the rigid body. Since a rigid body has six degrees of freedom $(D O F)$, there must be six equations describing the relation between forces and motion. This leads to the formulation of the Newton-Euler equations of motion for each segment:

$$
\begin{aligned}
\underline{\mathbf{F}}_{C M} & =m \frac{d^{2} \underline{\mathbf{p}}_{C M}}{d t^{2}} \\
\underline{\mathbf{M}} & =\frac{d\left(\mathbf{J}_{C M} \underline{\omega}\right)}{d t}
\end{aligned}
$$

Where $\underline{\mathbf{F}}_{C M}$ is the resulting external force acting on the center of mass $(C M)$ of the rigid body with mass $m$ and $\underline{\mathbf{p}}_{C M}$ is the position of $C M$. The first derivative with respect to time of $\underline{\mathbf{p}}_{C M}$ reflects the change of position in time, or the velocity of $C M$. Likewise, the second derivative of $\underline{\mathbf{p}}_{C M}$ (as in equation 4) reflects the change of velocity in time, or the acceleration of the center of mass. Any force, acting on the rigid body, can be divided in a force, acting on $C M$, and a moment of force $\underline{\mathbf{M}}$. The second vector equation in (4) resembles the first, as the resulting torque equals the product of rotation inertia tensor $\mathbf{J}_{C M}$ and angular acceleration (or the first time derivative of the angular velocity vector $\underline{\omega}$ (omega). However, unlike the mass $m, \mathbf{J}_{C M}$ is in general not constant in each coordinate system. This leads to considerable complications in 3-D; in 2-D on the other hand $\mathbf{J}_{C M}$ reduces to a single constant component.

Consider now the 2-D situation of a (foot) segment $A$ with mass $m$ and moment of inertia $J_{A}$ during the swing phase of walking. A local frame is attached to the center of mass with an angle $\theta$ to the global (inertial) frame $G$. The segment connects to another segment in joint $P$, the ankle joint (Fig. 7). Of course, the lower leg segment applies some connection forces on the foot in the ankle joint, expressed by the joint force $\underline{\mathbf{F}}_{P}$ and joint moment of force $M_{P}$. Finally, a gravity force applies to the foot, thus completing the free body diagram. When all vectors, which have both magnitude and direction, are expressed in global coordinates, Eq. (4) reduces to 3 scalar equations - scalars have magnitude only:

$$
{ }^{G} F_{P x}=m \cdot \frac{d^{2 G} r_{A x}}{d t^{2}}
$$




$$
\begin{aligned}
& { }^{G} F_{P y}-m \cdot g=m \cdot \frac{d^{2 G} r_{A y}}{d t^{2}} \\
& M_{P}+\left({ }^{G} r_{A P x} \cdot{ }^{G} F_{P y}-{ }^{G} r_{A P y} \cdot{ }^{G} F_{P x}\right)=J_{A} \cdot \frac{d \omega}{d t}
\end{aligned}
$$

Note that $g$ is the acceleration of gravity constant. The expression between brackets in the moment of force equation is the vector product of force and moment arm. Note also that the vector ${ }^{A} \underline{\mathbf{r}}_{A P}$ (expressed in the local frame $A$ ) is a constant but ${ }^{G} \underline{\mathbf{r}}_{A P}$ (The same vector but now expressed in global frame $G$ ) depends on the angle $\theta$ (and thus on time) as defined by equation (3). The vector ${ }^{G} \underline{\mathbf{r}}_{A}$ may be viewed as the translation vector in Eq. (1). With Eqs (1), (2) and (3), Eq. (5) may be expressed as three differential equations in the (time-dependent) degrees of freedom $r_{A x}, r_{A y}$ and $\theta$.

When the movement of the segment is known the second derivatives of the position and rotation coordinate may be calculated. Equation (5) may then be used to calculate the (unknown) joint forces and moments of force. Likewise, when additional known forces would be present, equations similar to Eq. (5) may be derived to compute the unknown forces at the ankle. For example, in an inverse analysis of the walking movement, the measured ground reaction forces are known. These would then contribute to the resulting forces and torques acting on the foot segment, and the equations of motion Eq. (4) are used to solve for the ankle force and torque. From these and the equations of motion of the shank segment, the knee forces are calculated, and so on.

\section{Application to human gait}

Bipedal walking is a complex movement, a balancing performance against the constant pull of gravity. Numerous definitions of walking are made, such as:

"In bipedal locomotion, man is continuously preventing a fall by placing one foot in front of the other."

"The two basic requisites of walking are the continuing ground reaction forces that support the body and the periodic movement of each foot from one position of support to the next in the direction of progression"

Apart from these formulations, walking can be quantified with a number of parameters. These are shown in the next sections: The step-parameters to describe the timing of the movement; kinematics for the movement itself (the joint rotations) and dynamics to describe the forces and moments of force that cause this movement.

The two ways to apply the equations of motion are usually referred to as the inverse dynamics and the direct (or forward) dynamics approach. In the inverse dynamics approach, the movement is assumed to be known and the forces and moments of force, needed to bring about that movement, are calculated (e.g. Koopman [9]). Inverse dynamics is applied in gait analysis, the equations of motion are usually derived with the Newton-Euler formulation. The estimated internal forces can be further processed in muscle models to estimate the distribution of muscle forces, which allows for a validation with measured EMG patterns.

In the direct dynamics approach, the movements of the segments are calculated by integrating the equations of motion, usually based on a Lagrangian formulation. This is only possible when the joint moments of force are known or assumed to be zero. The latter is the case in ballistic walking [10]. The joint moments of force can be found by trial and error such that a normal walking pattern results [12], from estimations of the muscle forces [11], as the result of an inverse dynamics model or by optimization techniques. This direct dynamics modeling will not be discussed further. 


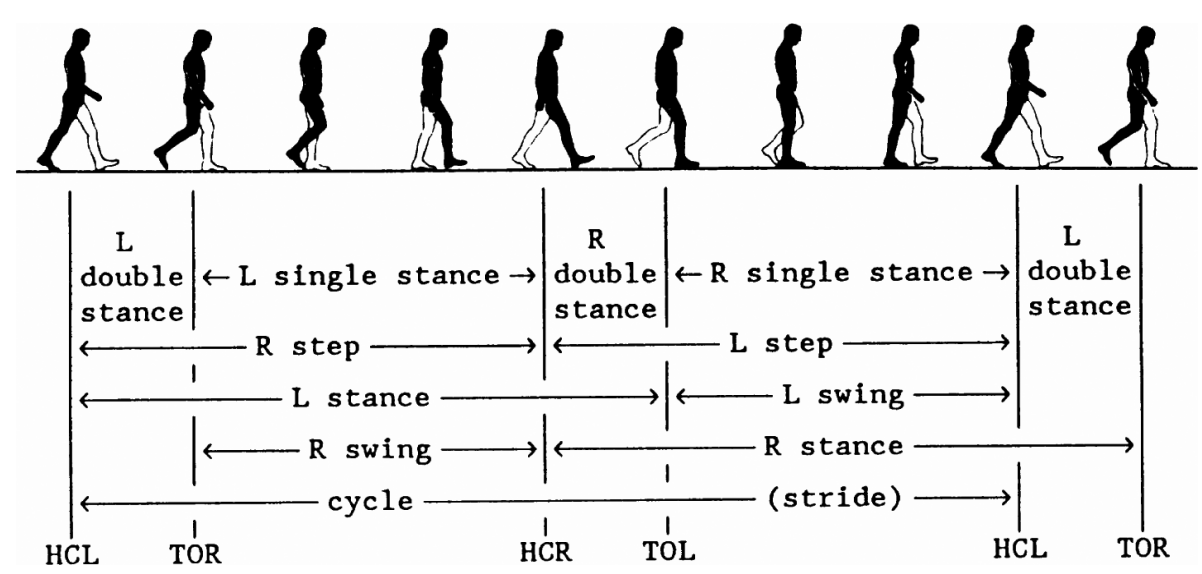

Fig. 8 . The walking cycle.

\subsection{Step-parameters}

The step-parameters are used for a general characterisation of the walking pattern. They are determined by the points of heel contact (HC) and toe-off (TO) of the left and right feet. For a walking cycle beginning with left heel contact (HCL), this is followed by right toe-off (TOR), right heel contact (HCR) and left toe-off (TOL). A cycle is completed with HCL again (Fig. 8).

These points divide the walking cycle in four different phases. For the left leg, the stance phase is from HCL to TOL and the swing phase (or the single stance phase of the right leg) is from TOL to HCL. There are two double stance phases where both feet are on the floor. The left double stance phase is from HCL to TOR. One stride consists of two steps: The left step is usually defined from HCR to HCL.

For the step-parameters, a distinction is made between the time-parameters and the distance-parameters. With the cycle beginning at $\mathrm{HCL}\left(t_{H C L}=0\right)$, four other time-parameters will suffice to define the points of $\mathrm{HC}$ and TO. These may be normalized with the stride or cycle time $T$ to make these time parameters dimensionless for comparison purposes. Likewise, the distance parameters can be made dimensionless with the stride length $S$. For symmetrical walking, the step-parameters are not independent any more.

Two step-parameters are derived from $S$ and $T$, which are the average forward velocity $v$ and the step ratio $r$. These are calculated from:

$$
\begin{aligned}
& v=S / T \\
& r=1 / 4 S \cdot T
\end{aligned}
$$

The step ratio is usually defined as the step length divided by the step frequency. In contrast to this, definition Eq. (7) does not depend on differences between right and left leg for asymmetrical walking. The reason for introducing the step ratio is that this parameter is shown to be reasonably constant for a wide range of walking velocities and for different subjects [13]. For normal walking, $r$ ranges from 0.39 to $0.44 \mathrm{~m} \cdot \mathrm{s}$ for men and from 0.34 to $0.40 \mathrm{~m} \cdot \mathrm{s}$ for women [7,15]. For a constant $r, S$ and $T$ are determined by the forward velocity only:

$$
\begin{aligned}
& T=2 \sqrt{r / v} \\
& S=2 \sqrt{r \cdot v}
\end{aligned}
$$



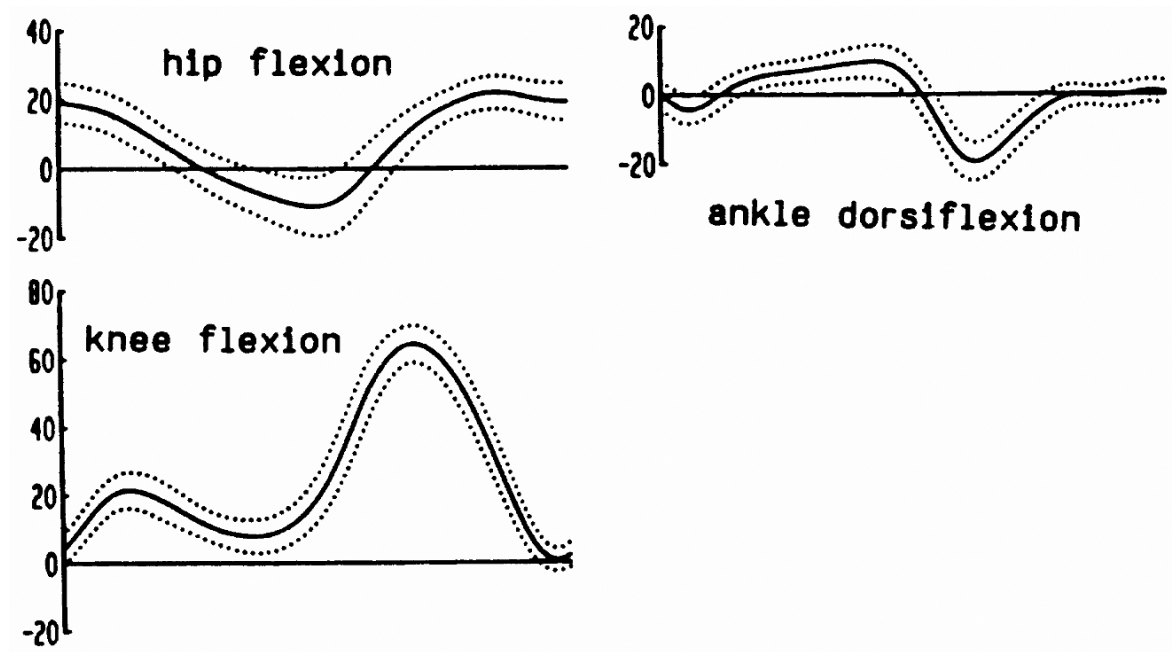

Fig. 9. Joint rotations in the sagittal plane for a cycle beginning with heel contact (in degrees). Hip flexion, knee flexion and ankle dorsiflexion; average values for 19 subjects with measuring deviations. From Winter (1991) [16].

\subsection{Kinematics}

A more detailed description of the walking movement is obtained when the joint rotations are measured at each point of time of the walking cycle. The hip, knee and ankle flexion are well documented in the literature, so measured data can be compared with "standard" data. As an example, the rotations that are shown in Fig. 9 are average values for normal walking, measured by Winter [16].

The other rotations that also contribute to the walking movement (e.g. hip adduction, pelvic rotations) are less well documented; accepted average values have yet to be established. The kinematics of the walking movement could also be described with the displacements of the joints as time functions or with a combination of displacements and rotations. The choice of one of these possibilities mostly depends on the measuring system that is available.

Note that a joint angle (e.g. hip angle) describes the orientation of one bone relative to another bone, or more precisely, the orientation of one local frame with respect to the other local frame of two connecting rigid bodies. A segment angle, on the other hand (e.g. the foot angle) describes the orientation of a local frame with respect to the global frame.

\subsection{Kinetics}

An alternative description of the walking movement is provided with the internal and external forces and moments of force, acting on the body segments. Of these forces only the ground reaction forces can be measured directly. The average ground reaction forces, as measured by Winter [16], are shown in Fig. 10.

Note that the average vertical ground reaction force should equal body weight. Variations from this average must result from accelerations of the total body centre of mass, according to Eq. (4).

The joint moments of force are determined by applying the Newton-Euler equations of motion Eq. (4). This always implies that some modeling assumptions, concerning the mechanical properties of the human body, have to be made. The joint moments of force are computed from the measured ground reaction forces and accelerations. Where the variation in the ground reaction forces between different subjects is 


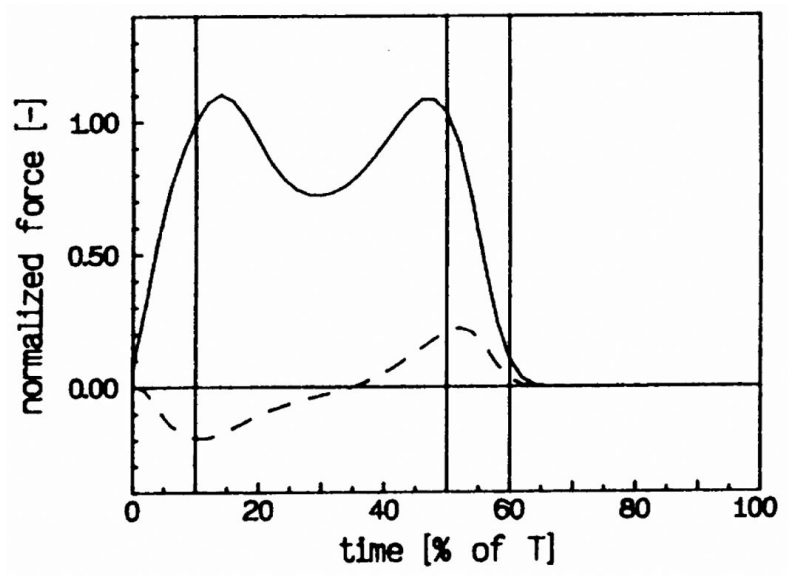

Fig. 10. Forward (- - ) and vertical (-) ground reaction forces, normalized with body width, ensemble average from 19 subjects. From Winter (1991) [16].

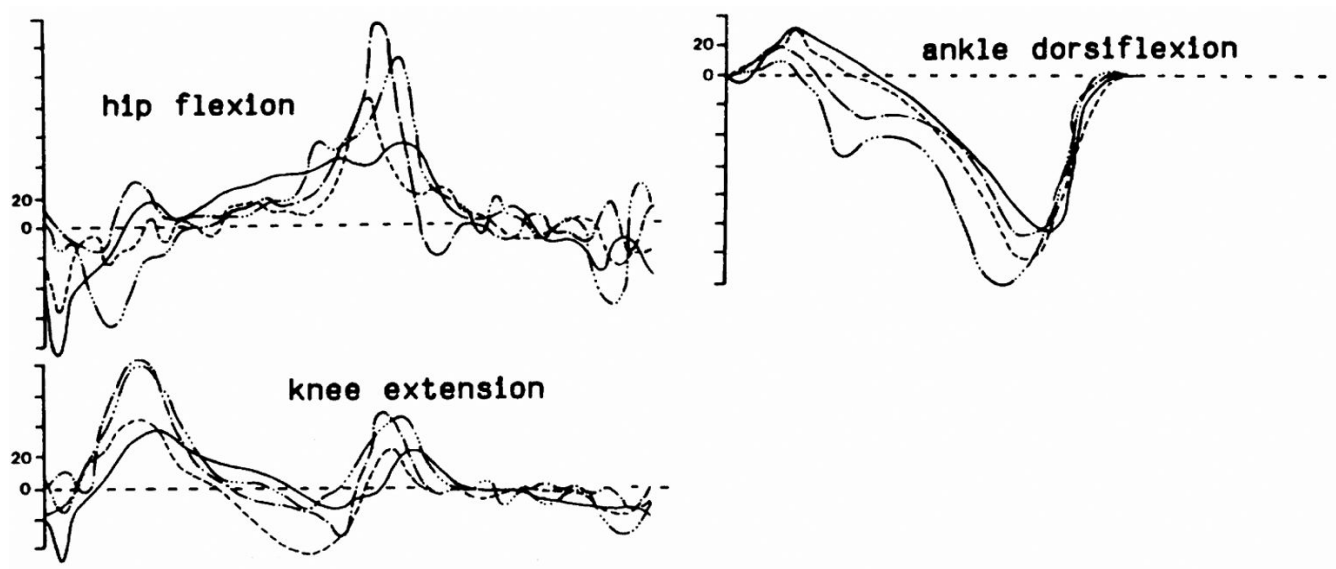

Fig. 11. Joint moments of force for four different subjects (in Nm). From Inman et al. (1981) [7].

reasonably small, due to the assumptions there may be a larger variation in the joint torques. Figure 11 shows some of the joint moments of force, as determined for four different subjects [7]. The joint moments of force can be viewed as the net result of all muscular, ligament and frictional forces acting on the joint. For example, the push-off with the calf musculature is clearly visible in the ankle torque of Figure 11. For some applications, the muscular forces can be related to electromyographic (EMG) signals, which may validate the modeling assumptions.

\subsection{Energy expenditure}

A distinction is usually made between mechanical energy and metabolic energy expenditure. Mechanical energy can only be determined by modeling and is based on computed kinetic and potential energies of segments or on joint powers.

Metabolic energy expenditure is measured by oxygen uptake during walking and is usually expressed in energy per unit time $\left(E_{w}\right)$ or energy per unit distance walked $\left(E_{m}\right)$. Ralston [14] first showed that $E_{w}$ 

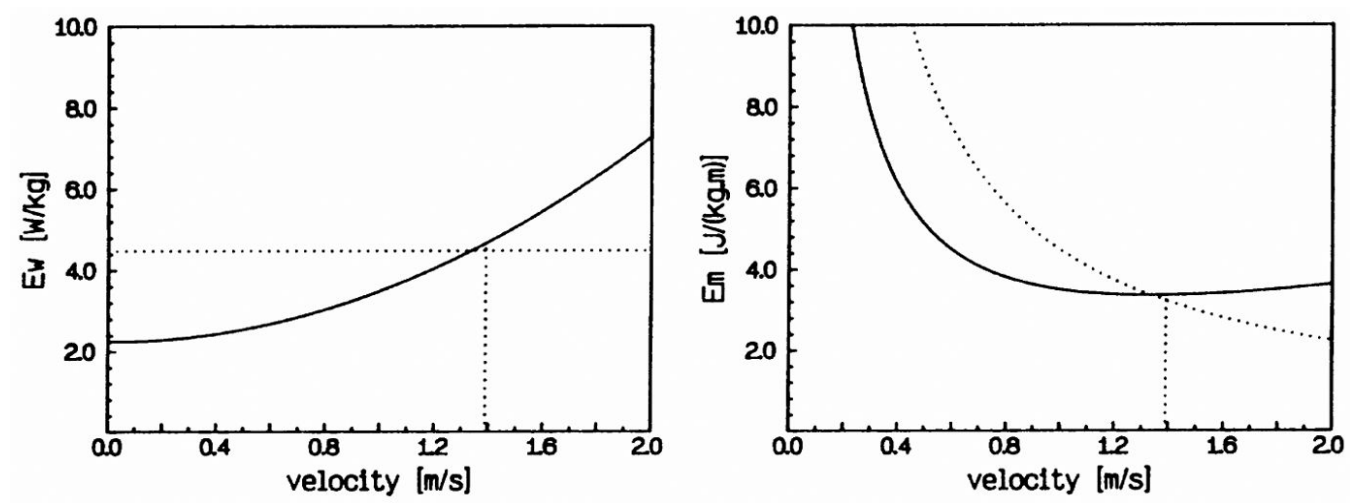

Fig. 12. Metabolic energy expenditures $\mathrm{E}_{w}$ and $\mathrm{E}_{m}$ for normal walking at different velocities.

is proportional to the square of the walking velocity:

$$
E_{w}=a+b v^{2}
$$

This relation is confirmed by various investigators [7]. When $E_{w}$ is expressed in watt per kg body mass and $v$ is in $\mathrm{m} / \mathrm{s}$, the experimental values for the constants $a$ and $b$ are $a=2.24 \mathrm{~W} / \mathrm{kg}$ and $b=1.26 \mathrm{~Hz}$. $E_{m}$ is defined by

$$
E_{m}=\frac{E_{w}}{v}=\frac{a}{v}+b \cdot v
$$

$\mathrm{E}_{w}$ and $\mathrm{E}_{m}$ are shown in Fig. 12.

The optimal velocity $v_{\text {opt }}$ is defined as the velocity where $E_{m}$ is minimal. Differentiating Eq. (11) with respect to $v$ and equating to zero yields $v_{\text {opt }}=1.33 \mathrm{~m} / \mathrm{s}$. The comfortable walking velocity $v_{\text {com } f}$ is the velocity a person tends to adopt in a natural walk. $v_{\text {comf }}$ is found to have an average value of 1.39 $\mathrm{m} / \mathrm{s}$ [7], which differs from

$v_{\text {opt }}$ by only $4 \%$. Inman et al. called this an example of a fundamental feature of human motor behavior: "In freely chosen rate of activity, a rate is chosen that represents minimal energy expenditure per unit task". In the case of walking a speed is adopted, with a specific stride length $S$ and cycle period $T$, such that each meter is covered as cheaply as possible. The energy expenditure $E_{m}$ can in this sense be interpreted as inversely proportional to the efficiency of walking.

In Fig. 12 is seen that $E_{m}$ varies only little for a wide range of velocities: The sensitivity of the efficiency to the velocity is small in this range. When the velocity is enforced, for example in a treadmill, the stride length, cycle period and joint rotations can still be chosen freely. The choice is such that the efficiency of the movement is maximized.

\subsection{Work balance}

By applying the equations of motion to the moving segments model in an inverse dynamics approach, the internal forces and moments of force are calculated. The product of the joint moment of force and the angular velocity equals the power output at the joint (Fig. 13). A positive power reflects energy generation; a negative power reflects energy dissipation at the joint. On a muscular level, this is comparable to concentric (i.e. the muscle shortens while pulling) and eccentric (i.e. the muscle lengthens while pulling, force opposite to movement) muscle contractions respectively. These joint powers finally 
Table 1

\begin{tabular}{ccccc}
\hline Joint $\mathrm{j}$ & \multicolumn{4}{c}{ Normal walking [J/cycle] } \\
\cline { 2 - 5 } & $W_{j}^{+}$ & $W_{j}^{-}$ & $W_{j}^{+}+W_{j}^{-}$ & $W_{j}^{+}-W_{j}^{-}$ \\
\hline R hip & 18 & -14 & -4 & 32 \\
L hip & 18 & -14 & -4 & 32 \\
R knee & 17 & -32 & -15 & 49 \\
L knee & 17 & -32 & -15 & 49 \\
R ankle & 13 & -3 & 10 & 16 \\
L ankle & 13 & -3 & 10 & 16 \\
total & 96 & -98 & -2 & 194 \\
\hline
\end{tabular}

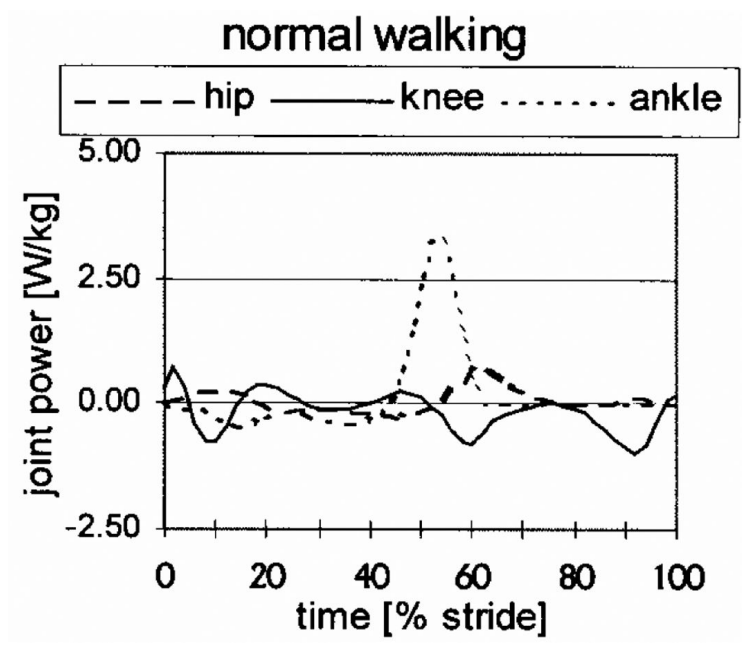

Fig. 13. Joint powers (in W/kg). Average values for 19 subjects. Obtained from Winter (1991) [16].

result in an increase (or decrease) of the mechanical energy (kinetic or potential) of the segments. However, since walking is a cyclic movement, the total power output of all the joints together in one cycle must equal zero for level walking. If for example the power output of one cycle would be positive, than the total mechanical energy of the segments model would be increased during one cycle. An increase of kinetic energy or walking speed is contradictory to the assumption of a cyclical movement; an increase of potential energy is only possible for walking uphill.

To be able to tell something about the work done at the joints, usually a distinction is made between the positive and the negative work $\left(W_{j}^{+}\right.$and $\left.W_{j}^{-}\right)$at joint $j$ with power $P_{j}$ :

$$
\begin{array}{ll}
W_{j}^{+}=\int_{0}^{T} P_{j}^{+} d t ; & P_{j}^{+}= \begin{cases}P_{j} & \left(P_{j}>0\right) \\
0 & \left(P_{j} \leqslant 0\right)\end{cases} \\
W_{j}^{-}=\int_{0}^{T} P_{j}^{-} d t ; & P_{j}^{-}= \begin{cases}0 & \left(P_{j} \geqslant 0\right) \\
P_{j} & \left(P_{j}<0\right)\end{cases}
\end{array}
$$

This definition results in a work balance as shown in Table 1. From the table it is obvious which joints in general generate energy and which joints dissipate energy. In most cases, the hip joints generate as much as they dissipate, the knee joints are mainly dissipaters (in the beginning of the stance phase to accept 
body weight) and the ankle joints are mainly generators (at the end of the stance phase to push off, also visible in Fig. 12). The total work for all joints during one cycle should equal zero. In Table 1, the total $W_{j}^{+}+W_{j}^{-}$equals $-2 \mathrm{~J} /$ cycle. This value reflects some inaccuracies of the calculation, especially the fact that the properties of the segments model do not perfectly match the properties of the human body.

The last column shows the absolute amount of mechanical work done. When it is assumed that monoarticular muscles do all the work, no energy is stored in passive structures, and the efficiency equals 1 for concentric contractions and -1 for eccentric contractions, this number can be related to the metabolic energy consumption. The metabolic energy consumption for a person of $80 \mathrm{~kg}$ with a stride length of $1.5 \mathrm{~m} /$ cycle is about $3.4 \times 80 \times 1.5=408 \mathrm{~J} /$ cycle (see Fig. 11 ).

About half this amount is needed for basal metabolism, the energy consumption at zero velocity. This leaves about $200 \mathrm{~J} / \mathrm{cycle}$ spendet on walking alone. This value is close to the estimated mechanical work (Table 1).

However, if more realistic values for the efficiencies are assumed (i.e. 0.3 and 1.0 for concentric and eccentric contractions respectively, see McMahon [10], the total mechanical work predicts a metabolic energy consumption of $418 \mathrm{~J} /$ cycle. This implies that energy transfer between joints through biarticular muscles and energy storage in passive structure are important mechanisms to reduce the over-all metabolic energy consumption. The work balances are especially useful when analyzing situations that deviate from normal.

\section{References}

[1] R.A. Brand, R.D. Crowninshield, C.E. Wittstock, D.R. Pedersen, C.R. Clark and F.M. van Krieken, A model of lower extremity muscular anatomy, J Biomech Eng 104 (1982), 304-310.

[2] R.F. Chandler, C.E. Clauser, J.T. McConville, H.M. Reynolds and J.W. Young, Investigation of the inertial properties of the human body, Report DOT HS-801430, National Technical Information Service, Springfield Virginia 22151, U.S.A, 1975.

[3] C.E. Clauser, J.T. McConville and J.W. Young, Weight, volume, and center of mass of segments of the human body. Aerospace Medical Research Laboratory TR-69-70 (AD 710 622), Wright-Patterson Air Force base, Ohio, 1969.

[4] R. Fick, Handbuch der Anatomie und Mechanik der Gelenke (Handbook of Joint Anatomy and Mechanics), Gustav Fischer, Jena. 1911

[5] H. Goldstein, Classical Mechanics (second edition). Addison Wesley Publishing company, Reading, Massachusetts. ISBN 0-201-02969-3, 1980.

[6] H. Hatze, Quantitative analysis, synthesis and optimization of human motion, Hum Movem Sc 3 (1981), 5-25.

[7] V.T. Inman, H.J. Ralston and F. Todd, Human Walking, Baltimore, Williams and Wilkins, 1981.

[8] B. Koopman, H.J. Grootenboer and H.J. Jongh de, An inverse dynamic model for the analysis reconstruction and prediction of bipedal walking, J Biomech 28 (1995), 1369-1376.

[9] H.F.J.M. Koopman, The three-dimensional analysis and prediction of human walking, Ph.D. Dissertation, University of Twente, Enschede, 1989.

[10] T.A. McMahon, Muscles, Reflexes, and Locomotion. Princeton University Press, Princeton, New Jersey, 1984.

[11] S.J. Olney and D.A. Winter, Predictions of knee and ankle moments of force in walking from EMG and kinematic data, J Biomech 18 (1985), 9-20.

[12] M.G. Pandy and N. Berme, A numerical method for simulating the dynamics of human walking, J Biomech 21 (1988), 1043-1051.

[13] R.H. Rozendal, P.A.J.B.M. Huijing, Y.F. Heerkens and R.D. Woittiez, Inleiding in de kinesiologie van de mens. (Introduction in the human kinesiology) Culemborg: Educaboek, 1990.

[14] H.J. Ralston, Energy-speed relation and optimal speed during level walking, Int Zeitschrift für angewandte Pysiologie 17 (1958), 277.

[15] R.L. Waters, B.R. Lunsford, J. Perry and R. Byrd, Energy-speed relationship of walking: standard tables, J Orthop Res 6 (1988), 215-222.

[16] D.A. Winter, The Biomechanics and Motor Control of Human Gait, Waterloo (Canada), University of Waterloo Press, 1991. 